Document downloaded from:

http://hdl.handle.net/10251/56298

This paper must be cited as:

Hellge, C.; David Gomez-Barquero; Schierl, T.; Wiegand, T. (2011). Layer-Aware Forward Error Correction for Mobile Broadcast of Layered Media. IEEE Transactions on Multimedia. 13(3):551-562. doi:10.1109/TMM.2011.2129499.

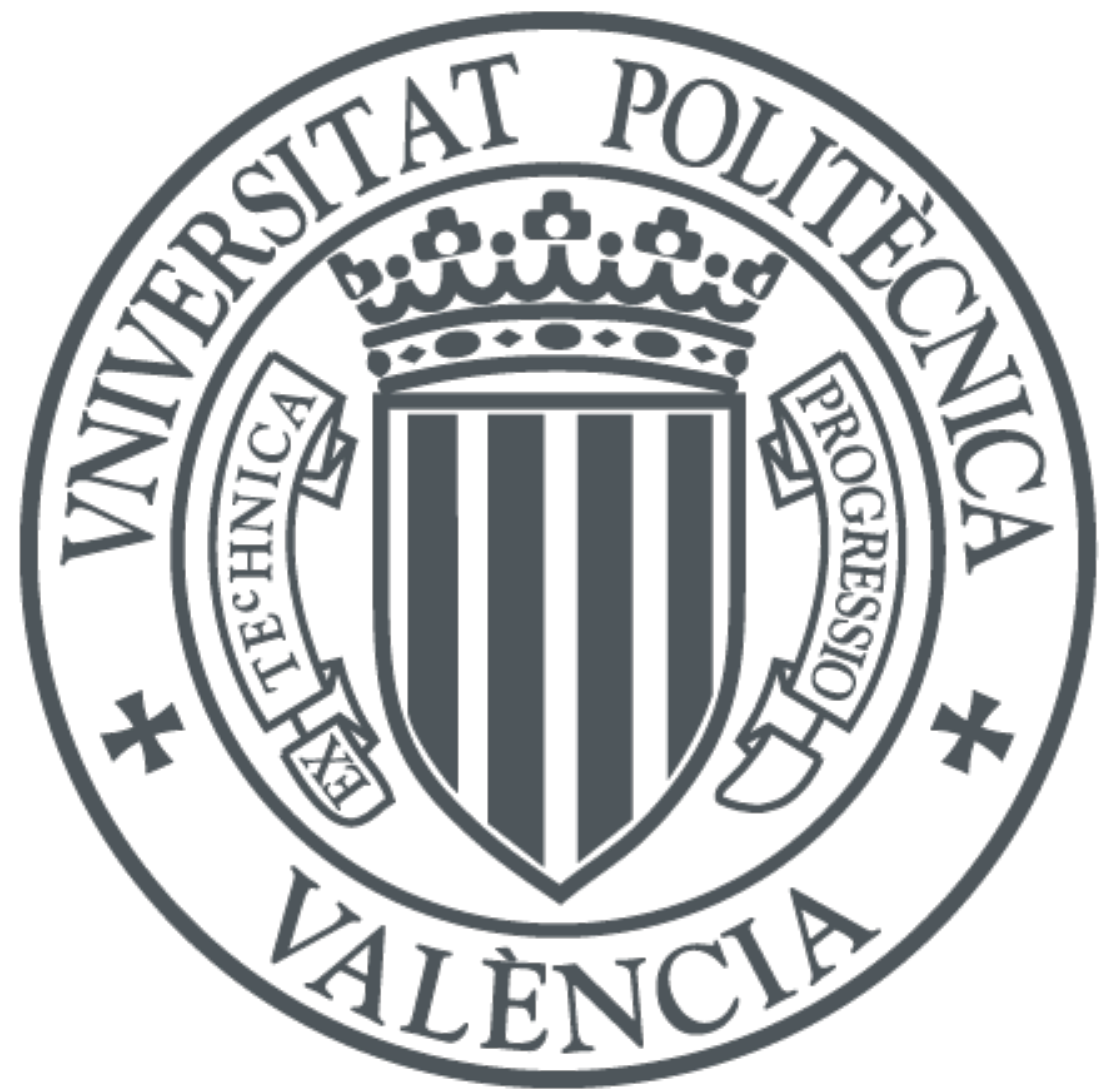

The final publication is available at

http://dx.doi.org/10.1109/TMM.2011.2129499

Copyright Institute of Electrical and Electronics Engineers (IEEE)

Additional Information 


\title{
Layer-Aware Forward Error Correction for Mobile Broadcast of Layered Media
}

\author{
Cornelius Hellge, David Gómez-Barquero, Thomas Schierl, Member, IEEE, and Thomas Wiegand, Fellow, IEEE
}

\begin{abstract}
The bitstream structure of layered media formats such as Scalable Video Coding (SVC) or Multiview Video Coding (MVC) opens up new opportunities for their distribution in Mobile TV services. Features like graceful degradation or the support of the 3D experience in a backwards-compatible way are enabled. Reason is that parts of the media stream are more important than others with each part itself providing a useful media representation. Typically, the decoding of some parts of the bitstream is only possible, if the corresponding more important parts are correctly received. Hence, unequal error protection (UEP) can be applied protecting important parts of the bitstream more strongly than others. Mobile broadcast systems typically apply forward error correction (FEC) on upper layers to cope with transmission errors, which the physical layer FEC cannot correct. Today's FEC solutions are optimized to transmit single layer video. The exploitation of the dependencies in layered media codecs for UEP using FEC is the subject of this paper. The presented scheme, which is called layer-aware FEC (LA-FEC), incorporates the dependencies of the layered video codec into the FEC code construction. A combinatorial analysis is derived to show the potential theoretical gain in terms of FEC decoding probability and video quality. Furthermore, the implementation of LA-FEC as an extension of the Raptor FEC and the related signaling are described. The performance of layer-aware Raptor code with SVC is shown by experimental results in a DVB-H environment showing significant improvements achieved by LA-FEC.
\end{abstract}

Index Terms-SVC, layered media, LA-FEC, Mobile TV, MVC, UEP

\section{INTRODUCTION}

$\mathrm{L}$ ayered media formats, such as Scalable Video Coding (SVC) [1] or Multiview Video Coding (MVC) [2] open up new opportunities for distributing Mobile TV services. Such services can benefit from features like graceful degradation behavior or introducing new services, e.g. providing additional

Manuscript received October 31, 2010.

Copyright (c) 2010 IEEE. Personal use of this material is permitted. However, permission to use this material for any other purposes must be obtained from the IEEE by sending a request to pubs-permissions@ieee.org.

C. Hellge and T. Schierl are with Multimedia Communications Group, Image Processing Department, Fraunhofer HHI, 10587 Berlin, Germany and with Image Communication Laboratory, Berlin Institute of Technology, 10623 Berlin, Germany (phone: +49-30-314-28772; e-mail: cornelius.hellge@hhi.fraunhofer.de; thomas.schierl@hhi.fraunhofer.de).

D. Gómez-Barquero is with Universidad Politécnica de Valencia, Valencia, 46022 Valencia, Spain and Multimedia Communications Group, Image Processing Department, Fraunhofer HHI, 10587 Berlin, Germany (e-mail: dagobar@iteam.upv.es).

T. Wiegand is with Image Processing Department, Fraunhofer HHI, 10587 Berlin, Germany and with Image Communication Laboratory, Berlin Institute of Technology, 10623 Berlin, Germany (e-mail: twiegand@ieee.org). higher resolution or 3D enhancements, in a backwardscompatible way. Layered media codecs are considered as a candidate technology in ongoing standardization on mobile broadcast services like e.g. SVC in DVB-NGH [3] or already adopted in ATSC-M [4]. Due to inter-layer prediction, parts of the media stream are more important than others. The loss of a certain quality layer affects all layers that depend on it. Therefore an efficient transmission of layered media requires a differentiation in robustness for the different layers of quality.

Forward error correction (FEC) is typically used in mobile broadcast systems to increase service robustness. FEC mechanisms can be categorized into those working at the physical layer or at any upper layer above it, such as the link or application layers [5]. On physical layer, typically LDPC [6] or Turbo codes [7] are applied. On upper layers, today's state of the art FEC solutions of mobile broadcast standards are Raptor code [8] or RaptorQ [9]. All these FEC algorithms are optimized for transmitting single layer video. The traditional FEC approach to achieve a more efficient delivery for multi-layer media is to apply unequal error protection (UEP) to the media stream, where more important layers get stronger FEC protection. This approach can already be implemented using the existing upper layer FEC schemes within DVB (DVB-H [10] or DVB-SH [11]) or 3GPP (MBMS [12]) by applying different code rates to the different video layers. On the physical layer, UEP can be implemented by applying hierarchical modulation [13] or different modulation and coding for the different video layers [14]. However, when UEP is done in such a way that both streams are independent, the referencing video layer (enhancement layer) is unusable if the referenced video layer (base layer) is lost.

With traditional UEP, the FEC parity data is generated separately for each layer. Several protection schemes have been proposed, which benefit in performance by considering the layered characteristic by integrating the UEP behavior within the FEC algorithm [15] - [23]. The Layer-Aware FEC (LA-FEC) [27] - [30] follows a similar approach. But instead of changing the basic FEC algorithm, it extends existing FEC algorithms towards improved decoding capabilities in case of dependent video layers. The basic FEC algorithm is not modified. Thereby preserving the optimized correction performance and easing backwards compatible introduction into existing systems. The LA-FEC scheme can be applied to the physical layer or upper layer FEC. In this paper we focus on upper layer FECs.

The rest of the paper is organized as follows. In Section II we discuss related work and the differences to this work. 
Section III gives a brief overview on layered video codecs and using FEC in mobile broadcast environments. In Section IV, the LA-FEC principle is explained, a combinatorial analysis is provided, and a discussion on implementation issues is given related to the integration into Raptor codes. The section further contains a discussion of the transport and signaling extensions required for the LA-FEC. In Section V exemplary simulation results are presented for an upper layer FEC integration with a layer-aware Raptor code in a DVB-H scenario.

\section{RELATED WORK}

Already in 1967, Masnick and Wolf proposed linear codes with UEP behavior [15] for the unequal protection of binary coded integer values. In 1972, the idea of two overlapping generator matrices was applied to cyclic codes by Kilgus and Gore [16] as well as to linear codes over Galois fields by Boyarinov and Katsman [17] in 1982. In 2006, Rahnvard et al. proposed an UEP-LDPC code [18], where parity symbols are generated across symbols of different importance classes. The selection of symbols depends on a probability distribution following its importance of the class. In [19], the same authors applied a similar scheme to LT-codes. Also in 2006, Bouabdallah and Lacan proposed to apply UEP erasure codes across temporal media coding dependencies within a single layer video stream [20]. In 2007, Bogino et al. [21] introduced a sliding window approach, which is based on a fixed size window following the chronological order of the data. This approach virtually increases the source block length which increases the FEC correction capability. In the same year, Sejdinovic et al. proposed the expanding window fountain (EWF) code [22][23]. EWF codes generate multiple windows over the source symbols, where windows expand according to the importance of the data. Encoding symbols are generated from a certain window, selected by a probability distribution. All these approaches introduce the UEP behavior within the FEC algorithm, which can be referred to as inner UEP FEC. In contrast to the mentioned inner UEP FEC approaches, the LAFEC approach can be seen as an outer UEP FEC, leaving the basic FEC algorithm untouched. I.e. the base layer processing is not changed at all. This eases the backwards compatible integration into existing systems, and preserves the high performance of state of the art FECs like Raptor [8] or RaptorQ [9].

\section{TECHNiCAL BACKGROUND}

\section{A. Layered Media Codecs}

Rate distortion efficient video codecs use prediction for exploiting statistical dependencies in the video signal, which introduces dependencies that typically also exist between packets. One important dependency structure is introduced by motion compensation, where a reference picture (e.g. from the past) is used to predict another picture [43]. Another set of dependency structures is introduced in layered video coding allowing for efficient scalability of the media data, such as Scalable Video Coding (SVC) [1] or Multiview Video Coding (MVC) [2], where in the simplest case a base layer is referenced by one enhancement layer (or enhancement view).

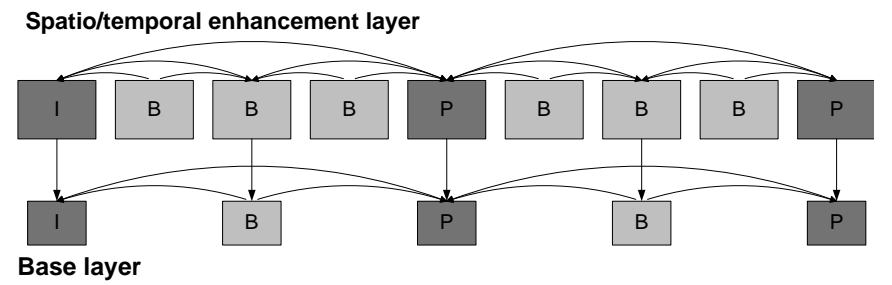

Figure 1: Dependencies within an SVC bitstream using hierarchical prediction and inter-layer prediction.

An enhancement layer can be further referenced by other enhancement layers potentially introducing multiple dependent layers. A loss of a picture in the base layer affects all pictures in the enhancement layer that reference the base layer, i.e. typically they cannot be decoded. Using layered media streams, each layer has a different level of importance in the decoding process of an access unit, representing a certain time instance of the video. If an access unit of a base layer gets lost, all referencing frames of the enhancement layers are affected as well.

\section{Scalable Video Coding (SVC)}

The Scalable Video Coding (SVC) extension of H.264/AVC allows for extracting different video representations from a single bitstream, where the different substreams are referred to as layers [1]. The base layer of SVC provides the lowest level of quality and is a H.264/AVC compliant bitstream to ensure backwards-compatibility with existing receivers. Each additional enhancement layer improves the video quality in a certain dimension. SVC allows up to three different scalability dimensions within one bitstream: temporal, spatial, and quality scalability. SVC utilizes different temporal and inter-layer prediction methods for gaining coding efficiency while introducing dependencies between quality layers of the SVC video stream. Figure 1 shows an exemplary coding structure, with the base layer and one enhancement layer at the same time enhancing temporal and the spatial resolution of the base layer. The arrows in the figure denote the coding dependencies between the different access units. In case of a lost access unit all referencing frames are affected too. E.g., if the I frame of the base layer gets lost, all other frames are affected.

A differentiation in robustness is in general beneficial for the transmission of the SVC format, where the base layer gets a stronger protection than the enhancement layers.

\section{Multiview Video Coding (MVC)}

Multiview video coding (MVC) is an amendment of the H.264/AVC standard that enables efficient encoding of sequences captured simultaneously from multiple cameras using a single video stream [2]. For MVC, the single-view concepts of H.264/AVC are extended in a way that a picture

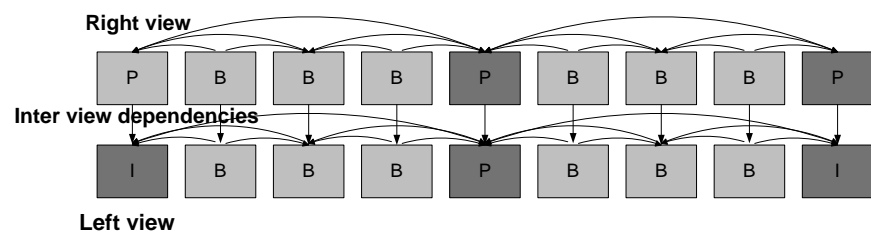

Figure 2: Dependencies within two views of a MVC stream, where the right view is encoded dependent on the base view. 
uses temporal reference pictures as well as inter-view reference pictures for predictive coding. Figure 2 illustrates an exemplary inter-view prediction structure using MVC. Due to the inter-view prediction in MVC, a differentiation in robustness is in general beneficial, like in SVC, where the base view gets a stronger protection than the enhancement view.

\section{B. Forward Error Correction (FEC)}

In mobile broadcast systems the transmission is typically designed to serve the worst-case user. Retransmissions of lost packets are generally not feasible due to a missing return channel in broadcast systems. Therefore, error correction is achieved using FEC mechanisms transmitting redundant data in form of additional repair data. This repair data allows the receivers for reconstructing the original data even if some data is not correctly received due to transmission errors. The error correction is "forward" in the sense that no feedback (return channel) from the receiver to the transmitter is required. FEC mechanisms can be categorized into those integrated at physical layer of a communication system and FEC mechanisms integrated at any layer above the physical layer, such as the link or application layers [24].

Physical layer FEC codes work at the bit level and are traditionally implemented as part of the radio interface of a wireless communication systems. Examples of physical layer FEC codes that are adopted in standards for mobile broadcasting are: convolutional codes in DVB-H [31], turbocodes in DVB-SH [44] or 3GPP [46], and Low-DensityParity-Check (LDPC) codes in DVB-T2 [45] or the future DVB-NGH system [3]. In contrast to physical layer FEC that corrects bit errors, upper layer FEC (UL-FEC) recovers packet losses and are categorized as block codes that work with fixed-size blocks (packets) of bits or symbols of a predetermined size performing erasure decoding. In UL-FEC, packets are considered either correct or lost. Examples of upper layer FEC codes in mobile broadcasting standards are Reed-Solomon in DVB-H [31] and Raptor codes in DVB-SH [44] and 3GPP [12].

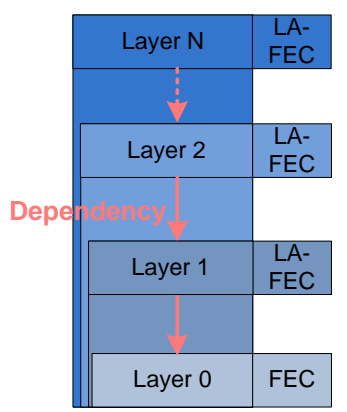

Figure 3: Generation of FEC data by LA-FEC across layers following dependency within the media stream.

\section{LAYER-AWARE FORWARD ERROR CORRECTION (LA-FEC)}

\section{A. General Description}

The basic idea of the Layer-Aware FEC (LA-FEC) approach is to extend the encoding process of the FEC algorithm across dependent video layers. The FEC processing of the base layer remains untouched, thereby still allowing the base layer to be decoded independently and preserving the correction capabilities of the original FEC algorithm. Due to the introduced connection from less important media layers within the FEC algorithm, the more important media layers are protected by additional repair data. This increases the error correction capabilities of the more important layers without adding additional repair data. The scheme in Figure 3 illustrates the cross layer FEC generation. While the base layer ("Layer 0") FEC generation process is not changed, the FEC data of "Layer 1" is generated across source symbols of "Layer 1" and "Layer 0", FEC data of "Layer 2" is generated across "Layer 2", "Layer 1", and "Layer 0" and so on up to the FEC data of "Layer N", which is generated across the source symbols of "Layer $\mathrm{N}$ " and all dependent media layers. As a generic FEC approach, LA-FEC can be integrated at any OSI layer (physical, link, or application layer), and to FEC codes like LDPC, Raptor, or RaptorQ, by simply extending the encoding process of the media enhancement layers over all dependent media layers.

To illustrate the principle of the LA-FEC approach we apply a simple FEC algorithm which generates parity bits by XOR combinations of source symbols (one bit per symbol). Figure 4 compares the encoding process, and Figure 5 the decoding process of standard FEC (ST-FEC) on the left side and LA-
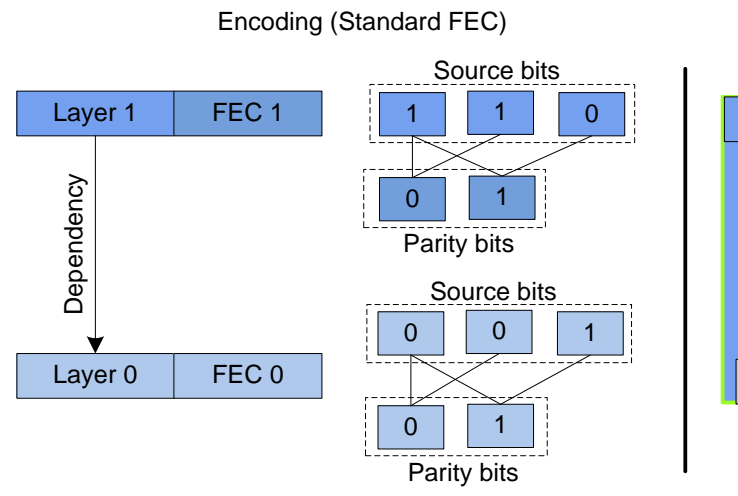

Encoding (LA-FEC)
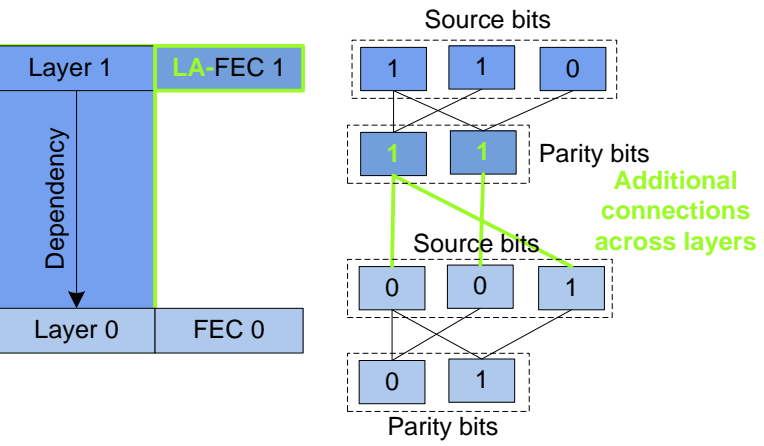

Figure 4: Encoding for ST-FEC (left) and LA-FEC (right). LA-FEC extends generation of parity bits across "Layer 0" symbols. 

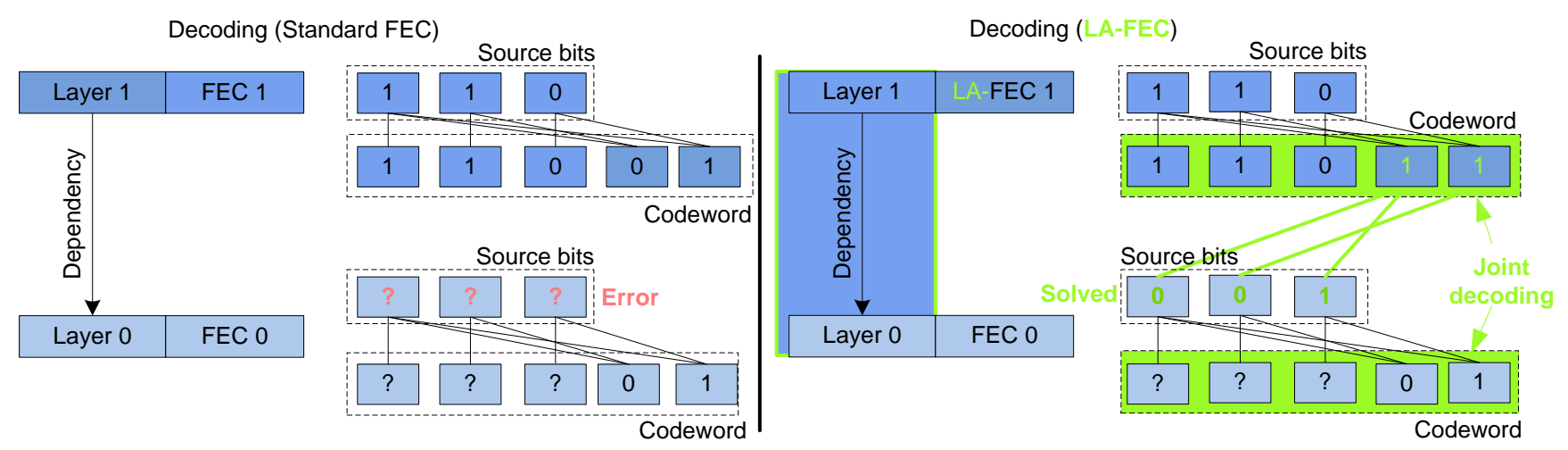

Figure 5: Decoding of ST-FEC (left) and LA-FEC (right). Using LA-FEC the parity bits of both layers can be used for a combined decoding.

FEC on the right side of each figure. LA-FEC modifications are marked in green. In the given example, which is based on an erasure channel (erroneous packets are treated as lost packets), there are two media quality layers, where "Layer 1" depends on "Layer 0" within the media stream. Each layer consists of three source bits and two parity bits.

With respect to the exemplary encoding process presented in Figure 4, the parity bits are computed by a simple XORing process of the source bits. Using ST-FEC, the XORing process is applied independently for each media layer, whereas using LA-FEC, the XORing process is extended across media layers following existing media coding dependencies. Hence, the parity bits of "Layer 1" are generated over the source bits of both layers, "Layer 0 " and "Layer 1". The "Layer 1" parity bits can further be used jointly with the parity bits of "Layer 0 " for error correction of both media layers. After FEC encoding, the source and parity bits of each media layer are combined to codewords. The codewords are in the example transmitted over an error prone channel.

In the decoding example in Figure 5, the codeword of "Layer 0 " is affected by three transmission errors labeled by "?". "Layer 1" is received error free. In case using ST-FEC, there are not enough parity bits within "Layer 0 " for successful FEC decoding. The source bits can therefore not be recovered. Although "Layer 1" codeword is correctly received, it cannot be used due to the missing media coding dependencies on "Layer 0". In contrast to that, if using the LA-FEC, the parity bits of "Layer 1" can be used jointly with the parity bits of "Layer 0" for also correcting "Layer 0". Since "Layer 1" is correctly received, there are overall four parity bits available for correction of the three source bits of "Layer 0". In the given example, both media layers can only be corrected with LA-FEC. It should be noted, that if using the LA-FEC, the enhancement layer cannot be corrected independently of the base layer. Therefore, the improvement in base layer protection comes at the expense of a reduced protection of the enhancement layer. Nevertheless, in cases where the base layer is lost, the enhancement layer data cannot be used in the media decoding process anyway due to missing media dependencies within the media stream. Therefore, LA-FEC does not perform worse than the ST-FEC in terms of media quality.

\section{B. Combinatorial Analysis of LA-FEC}

In this section the LA-FEC approach is analyzed towards its influence on the decoding probability of each media layer. The performance of LA-FEC in comparison to ST-FEC is shown by a combinatorial analysis based on an erasure channel model.

The conducted analysis is based on an example, illustrated in Figure 6, with two video layers, "Layer 0" and "Layer 1". Due to media coding dependencies, "Layer 1" directly depends on "Layer 0". Each layer consists of a certain amount of source symbols $k_{1}, k_{2}$ and a number of parity symbols $p_{1}, p_{2}$. All symbols of the two media layers $n=n_{0}+n_{1}$ are sent over a binary erasure channel. Transmission errors results in loss of a symbol. An ideal FEC code is assumed, where any $k$ source symbols can be corrected as soon as $r \geq k$ symbols have been received. The average decoding probability for each layer is calculated for each number of $r$ and all possible distributions of the lost symbols (loss constellations). In Figure 7 - Figure 9, $r$ is referred to as ratio of received packets, which means the percentage of received packets of the sent packets $n$, and can be calculated by $\frac{r}{n}$. Figure 6 depicts the example with $k_{0}, k_{l}=3$, $p_{0}, p_{1}=3, n=12$ and one exemplary loss constellation for $r_{0}=2$, $r_{1}=4$, and $r=6$.

The number of all possible loss constellations $|\Omega|$ for a number of received symbols $r$ can then be calculated by the binominal coefficient of the received packets $r$ choose $n$ sent packets as shown in (1).

$$
|\Omega|(r)=\left(\begin{array}{l}
n \\
r
\end{array}\right)=\frac{n !}{r !(n-r) !}
$$

The decoding probability of each media layer $L: 0 \mid l$ depends on the number of decodable loss constellations $\left|\Omega_{0 \mid 1}\right|$. For STFEC, the number of decodable combinations can be calculated by comparing the number of received symbols $r_{0 \mid I}$ and source symbols $k_{0 \mid 1}$. Thus, for ST-FEC, media layer is decodable if

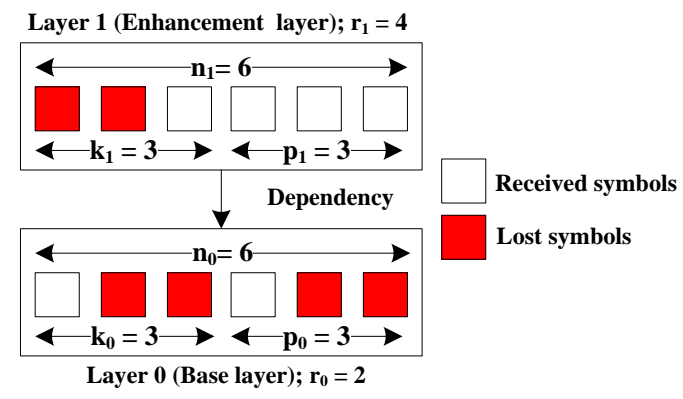

Figure 6: Toy example with two layers and $n=n_{0}+n_{1}=12$ transmitted and $r=r_{0}+r_{l}=6$ received symbols. The figure shows one exemplary distribution of lost symbols for $r_{0}=2$ and $r_{l}=4$. 
condition (2) is true.

$$
r_{0 \mid 1} \geq k_{0 \mid 1}
$$

The number of all constellations $\left|\Omega_{0 \mid 1}\right|$ fulfilling condition (2) for a given received number of symbols $r$ can be derived by equation (3).

$$
\left|\Omega_{0 \mid 1}\right|\left(r \mid r \geq k_{0 \mid 1}\right)=\sum_{x=\max \left(k_{0 \mid 1}, r-n_{1 \mid 0}\right)}^{\min \left(r, n_{0 \mid 1}\right)}\left(\begin{array}{c}
n_{0 \mid 1} \\
x
\end{array}\right)\left(\begin{array}{c}
n_{1 \mid 0} \\
r-x
\end{array}\right)
$$

For ST-FEC the decoding probability $P\left(\Omega_{0 \mid 1}\right)$ of each media layer can further be derived by equation (4).

$$
P\left(\Omega_{0 \mid 1}\right)(r)=\frac{\left|\Omega_{0 \mid 1}\right|\left(r \mid r \geq k_{0 \mid 1}\right)}{|\Omega|(r)}
$$

However, the media coding dependencies between media layers are not taken into account in (4). Taking such media coding dependencies into account, the decoding probability of the media layers is also affected by the decoding probability of the media layer it depends on. Thereby, the decoding probability of the enhancement layer $L=1$ depends on the number of loss constellations fulfilling condition (5).

$$
\left(r_{1} \geq k_{1}\right) \cap\left(r_{0} \geq k_{0}\right)
$$

According to this formula, the number of constellations giving a successfully decodable enhancement layer $\left|\Omega_{1 d e 0}\right|$ can be derived by equation (6). Note that for being able to decode both layers, condition $r \geq k_{0}+k_{l}$ must be true.

$$
\left|\Omega_{1 d e 0}\right|\left(r \mid r \geq k_{0}+k_{1}\right)=\sum_{x=\max \left(r-n_{1}, k_{0}\right)}^{\min \left(r-k_{1}, n_{0}\right)}\left(\begin{array}{c}
n_{0} \\
x
\end{array}\right)\left(\begin{array}{c}
n_{1} \\
r-x
\end{array}\right)
$$

and the probability $P\left(\Omega_{1 d e 0}\right)$ is calculated by equation (7).

$$
P\left(\Omega_{1 \text { de } 0}\right)(r)=\frac{\left|\Omega_{1 \mathrm{de} 0}\right|\left(r \mid r \geq k_{0}+k_{1}\right)}{|\Omega|(r)}
$$

In the case of LA-FEC, the additional FEC connections between dependent media layers influences the decoding probability of all media layers. The decoding probability for the base layer is increased by the probability that the enhancement layer receives more symbols than required for decoding of the enhancement layer symbols. Therefore, with LA-FEC, the condition for a successfully decodable base layer in (2) changes to the condition in (8)

$$
\left(r_{0} \geq k_{0}\right) \cup\left(r_{0}+r_{1} \geq k_{0}+k_{1}\right)
$$

and thereby the number of decodable constellations is increased, where the additional constellations $\Delta\left|\Omega_{0-L A}\right|$ can be calculated by (9). Note that there is only gain if both layers can be decoded, i.e. the condition $r \geq k_{0}+k_{l}$ is true.

$$
\Delta\left|\Omega_{0-L A}\right|\left(r \mid r \geq k_{0}+k_{1}\right)=\sum_{\mathrm{x}=\max \left(r-n_{1}, 0\right)}^{k_{0}-1}\left(\begin{array}{c}
n_{l} \\
x
\end{array}\right)\left(\begin{array}{c}
n_{l+1} \\
r-x
\end{array}\right)
$$

and the decoding probability $P\left(\Omega_{0-L A}\right)$ can be calculated by (10).

$$
P\left(\Omega_{0-L A}\right)(r)=\frac{\left|\Omega_{0}\right|\left(r \mid r \geq k_{0}\right)+\Delta\left|\Omega_{0-L A}\right|\left(r \mid r \geq k_{0}+k_{1}\right)}{|\Omega|(r)}
$$

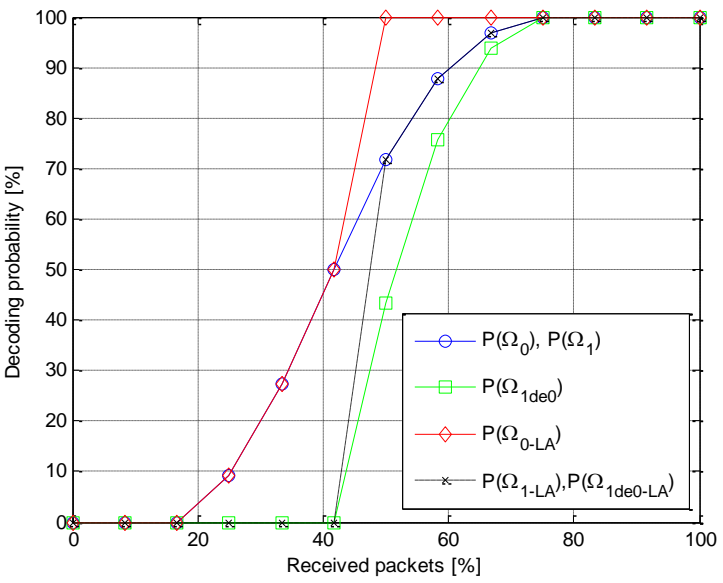

Figure 7: Decoding probability (cf. Table I) for "Layer 0" and "Layer 1" over the ratio of received packets for ST-FEC and LA-FEC.

On the other side, using LA-FEC without taking media coding dependencies into account, the sheer FEC decoding probability for the enhancement layer decreases since it can only be corrected if the base layer can also be corrected. Therefore, the condition for a successfully decodable enhancement layer without taking media coding dependencies into account changes from equation (2) to equation (11).

$$
\left(r_{1} \geq k_{1}\right) \cap\left(r_{0}+r_{1} \geq k_{0}+k_{1}\right)
$$

The number of non decodable constellations $\Delta\left|\Omega_{1-L A}\right|$ enabled by the LA-FEC can be determined by (12)

$$
\Delta\left|\Omega_{1-L A}\right|\left(r \mid k_{0}+k_{1}>r \geq k_{1}\right)=\sum_{\mathrm{x}=\max \left(r-n_{1}, 0\right)}^{\min \left(r-k_{1}, k_{0}-1\right)}\left(\begin{array}{c}
n_{0} \\
x
\end{array}\right)\left(\begin{array}{c}
n_{1} \\
r-x
\end{array}\right)
$$

and the decoding probability of the enhancement layer $P\left(\Omega_{1}\right)$ in equation (4) decreases for the case using the LA-FEC to $P\left(\Omega_{1-L A}\right)$ following (13).

$$
P\left(\Omega_{1-L A}\right)(r)=\frac{\left|\Omega_{1}\right|\left(r \mid r \geq k_{1}\right)-\Delta\left|\Omega_{1-L A}\right|\left(r \mid k_{0}+k_{1}>r \geq k_{1}\right)}{|\Omega|(r)}
$$

Taking media coding dependencies into account, the decoding probability of the enhancement layer $P\left(\Omega_{1 d e 0-L A}\right)$ is equal to $P\left(\Omega_{1-L A}\right)$, since all non decodable cases are already taken into account due to the dependencies introduced by the LA-FEC. Therefore equation (14) is true for the decoding probability $P\left(\Omega_{1 d e 0-L A}\right)$.

$$
P\left(\Omega_{1 d e 0-L A}\right)(r)=P\left(\Omega_{1-L A}\right)(r)
$$

All discussed decoding probabilities are summarized in Table I.

Table I: DECODING PROBABILITIES

\begin{tabular}{|l|c|c|c|c|}
\hline & $\begin{array}{c}\text { Base } \\
\text { layer }\end{array}$ & $\begin{array}{c}\text { Enh. } \\
\text { layer }\end{array}$ & LA-FEC & $\begin{array}{c}\text { Media } \\
\text { dependencies }\end{array}$ \\
\hline$P\left(\Omega_{0}\right)$ & $\mathrm{X}$ & - & - & - \\
\hline$P\left(\Omega_{1}\right)$ & - & $\mathrm{X}$ & - & - \\
\hline$P\left(\Omega_{1 d e 0}\right)$ & - & $\mathrm{X}$ & - & $\mathrm{X}$ \\
\hline$P\left(\Omega_{0-L A}\right)$ & $\mathrm{X}$ & - & $\mathrm{X}$ & - \\
\hline$P\left(\Omega_{1-L A}\right)$ & - & $\mathrm{X}$ & $\mathrm{X}$ & - \\
\hline$P\left(\Omega_{1 d e 0-L A}\right)$ & - & $\mathrm{X}$ & $\mathrm{X}$ & $\mathrm{X}$ \\
\hline
\end{tabular}


The decoding probability for the different cases is shown in Figure 7 over the ratio of received symbols. For ST-FEC, taking the media coding dependency into account, the enhancement layer decoding probability $P\left(\Omega_{1 \text { de0 }}\right)$ compared to the base layer decoding probability $P\left(\Omega_{0}\right)$ is significantly reduced. Using LA-FEC instead, the base layer decoding probability $P\left(\Omega_{0-L A}\right)$ is increased from $43 \%$ to $75 \%$ of ratio of received packets and already reaches $100 \%$ decoding probability after reception of $50 \%$ of all transmitted symbols. On the other side, the sheer enhancement layer FEC decoding probability $P\left(\Omega_{1-L A}\right)$ decreases compared to $P\left(\Omega_{1}\right)$ due to the additional FEC dependencies introduced by LA-FEC. However, taking the media coding dependency into account using the LA-FEC, even the enhancement layer shows a higher decoding probability $P\left(\Omega_{1 d e 0-L A}\right)$, which is due to the higher FEC decoding probability of the base layer.

The presented decoding probabilities are calculated for one exemplary distribution of parity data within the toy example with $p_{1}, p_{2}=3$, which is referred to as equal error protection (EEP). We further analyze the influence of the distribution of the parity symbols among the media layers on the decoding probability. The distribution of parity symbols is indicated by the code rate $(\mathrm{CR}) c_{l}$ for each layer $l$, which is calculated by the number of source symbols $k_{l}$ of layer $l$ to the number of transmitted symbols per layer $n_{l}=k_{l}+p_{l}$ following equation (15).

$$
c_{l}=\frac{k_{l}}{n_{l}}
$$

For the enhancement layer we assume a successful decoding only if the base layer can be decoded as well. The results for both media layers are shown in Figure 8.

For ST-FEC, the base layer decoding probability (left plot) solely depends on its code rate $c_{0}$. It should be noted here that the selection of the code rate distribution for layered media is given by the target application and needs to take the decoding probability of both media layers into account. For the base layer decoding probability (left plot), the best performance is given by applying all protection symbols to the base layer with setting $C R(0.33 / 1.00)$ and the worst performance when applying all protection symbols to the enhancement layer $C R(1.00 / 0.33)$. Also in the case using LA-FEC, the best base layer performance can be achieved by setting $C R(0.33 / 1.00)$. The influence of the enhancement layer code rate when using LA-FEC on the decoding probability of the base layer can be

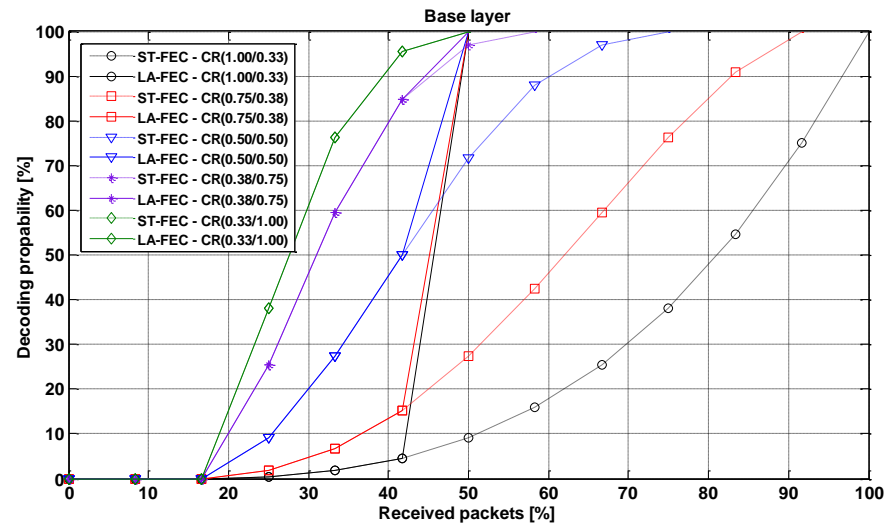

easily seen in the plot. Independent of the code rate distribution among layers, the maximum decoding probability is reached by receiving $50 \%$ of all symbols and all code rate distributions show an additional gain for lower reception ratios. It can be noticed, that the gain introduced by LA-FEC increases with a stronger enhancement layer protection.

The enhancement layer performance (right plot) is calculated taking the media dependency to the base layer into account (comp. Figure 7) . Therefore, the performance for ST-FEC and LA-FEC is affected by both, the base layer and the enhancement layer robustness. It is obvious, that the best performing base layer code rate distribution $C R(0.33 / 1.00)$ gives the worst results for the enhancement layer. Shifting all protection to the enhancement layer $C R(1.00 / 0.33)$ gives the best performance for LA-FEC but the worst performance for ST-FEC. Again, the gain over ST-FEC achieved by the LAFEC depends on the amount of protection symbols within the enhancement layer.

To analyze the LA-FEC performance, it is important to consider the video quality in terms of PSNR, which is affected by the FEC decoding probabilities of both media layers. The results shown in Figure 9 are based on the following assumptions: A non-decodable constellation, which would result in a freeze of the video, gives a video quality in terms of peak-signal-to-noise-ratio (PSNR) of $14 \mathrm{~dB}$. It should be noted that values of PSNR significantly below $20 \mathrm{~dB}$ are typically not acceptable from the user point of view. A constellation, where the base layer is decodable results in a PSNR value of about $30 \mathrm{~dB}$ and an additional decodable enhancement layer results in a PSNR of $35 \mathrm{~dB}$. Figure 9 shows the average PSNR over the ratio of received packets. The plot shows that LAFEC outperforms ST-FEC for all code rate distributions except for $C R(0.33 / 1.00)$, which performs equal, and never performs worse. Shifting all protection to the enhancement layer $C R(1.00 / 0.33)$ allows to reach the highest quality at $50 \%$ symbol reception rate. However, such a code rate distribution would put a hard burden on receiving the base layer only. It is also interesting to see, that LA-FEC allows reaching performance areas, which are not possible with ST-FEC independent of the code rate distribution. The presented theoretical results lack in a realistic channel model for mobile broadcast which typically shows a tendency towards burst errors, which have not been part of the experiments conducted in this section. More realistic performance measurements are

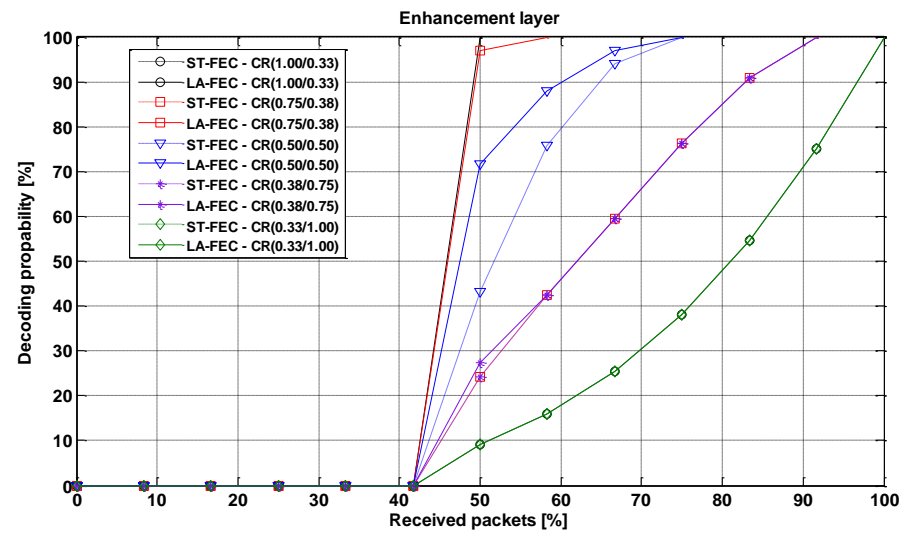

Figure 8: Decoding probability of base and enhancement layer using different parity distributions $C R\left(c_{d} / c_{1}\right)$ for ST-FEC and LA-FEC taking media dependencies into account. 


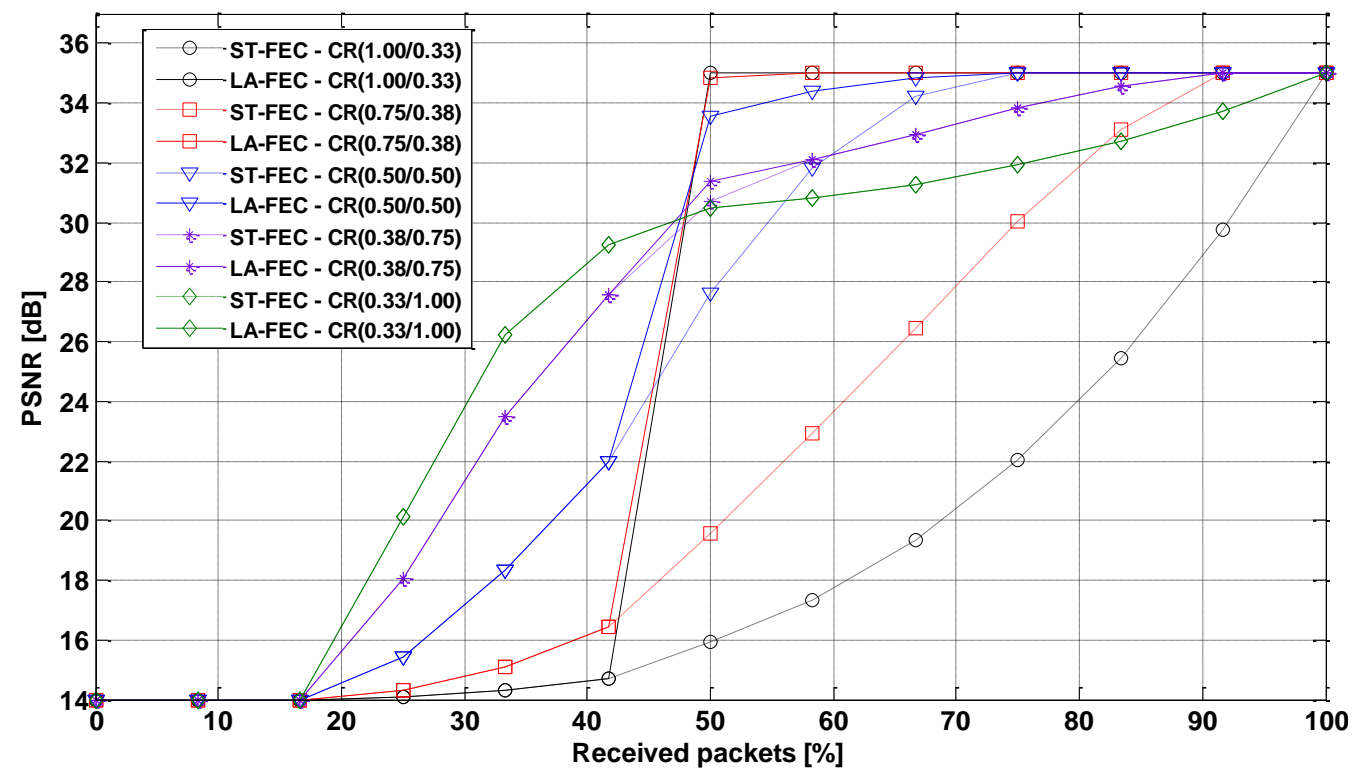

Figure 9: Average PSNR using different parity distributions $C R\left(c_{0} / c_{1}\right)$ for ST-FEC and LA-FEC assuming PSNR $14 \mathrm{~dB}$ for a non decodable constellation, $30 \mathrm{~dB}$ for a decodable base layer, and $35 \mathrm{~dB}$ for decodable enhancement layer.

given in Section $\mathrm{V}$.

\section{Implementation of Layer-Aware Raptor code}

The LA-FEC scheme can be applied on both, physical layer FEC, as similarly shown for LDPC in [40], and upper layer FECs [8][9]. This paper focuses on an exemplary integration of the LA-FEC to an upper layer FEC. The here considered upper layer FEC is the Raptor FEC.

The application of the LA-FEC to the Raptor code has already been presented in [27]. This section gives a brief summary of [27]. The required extensions for a systematic Raptor code as, e.g. specified in 3GPP MBMS [12] and or DVB-SH (for MPE-iFEC) [11]. A full specification based on [26] can be found in the Annex D of the DVB Upper layer FEC overview [5], which discusses the possibility to integrate LA-FEC within DVB. Note that the extension could be applied in a similar way to the more efficient RaptorQ FEC [9] codes.

Raptor codes are in general one of the first known classes of fountain code with linear time encoding and decoding [8]. In preparation of the encoding, a certain amount of data is collected within a source block. The data of a source block is further divided in $k$ source symbols $(S S s)$ of a fixed symbol size. Figure 10 illustrates the Raptor encoding process for a single media "Layer 0", which consists of two encoding steps [26]. In the first step, a fixed rate 'precode' step, here typically any erasure code like, e.g. LDPC, can be applied on the $S S s_{0}$ to generate the so called precoding symbols $\left(P S s_{0}\right)$. The values of the $P S s_{0}$ are determined by the matrix $G_{S y s 0}$, which consists of the precode matrix $G_{P 0}$, the identity matrix $I$, and the LT matrix $G_{L T O}\left[0: k_{0^{-}}-1\right]$, where the latter is identical to the first $k_{0}$ rows of $G_{L T O}\left[0: n_{0}-1\right]$ in the second encoding process. The values within the brackets denote the number of rows. The integration of the matrix $G_{L T O}$ assures, that the first $k_{0}$ encoding symbols $\left(E S s_{0}\right)$ after LT encoding are identical to the $S S s_{0}$. After finalizing the first step, the $P S s_{0}$ are forwarded to the second step. The fountain of $n_{0}$ encoding symbols $E S s_{0}$ are calculated by XORing $P S s_{0}$ following the connection given by the LT code and illustrated by the $G_{L T O}\left[0: n_{0^{-}} 1\right]$ matrix. Note, that also with LA-FEC, the generation of base layer $E S s_{0}$ follows the original Raptor process.

For the enhancement "Layer 1", the LA-FEC approach needs to be integrated into the Raptor coding process [26], which requires on one hand the extension of the $G_{L T}$ matrix of the LT-encoding step of the PSs in the dependent media layers and on the other hand the extension of the $G_{L T}$ matrix of the precoding process to preserve the systematic behavior of the code. Figure 11 shows the required extensions, highlighted in green, for generation of $\mathrm{ESs}_{1}$.

In the first encoding step, the generation of the matrices $G_{p l}$, $I$, and $G_{L T I}\left[0: k_{1}-1\right]$ is identical to the related matrices shown in Figure 10. LA-FEC requires the extension of the matrix $G_{S y s}$

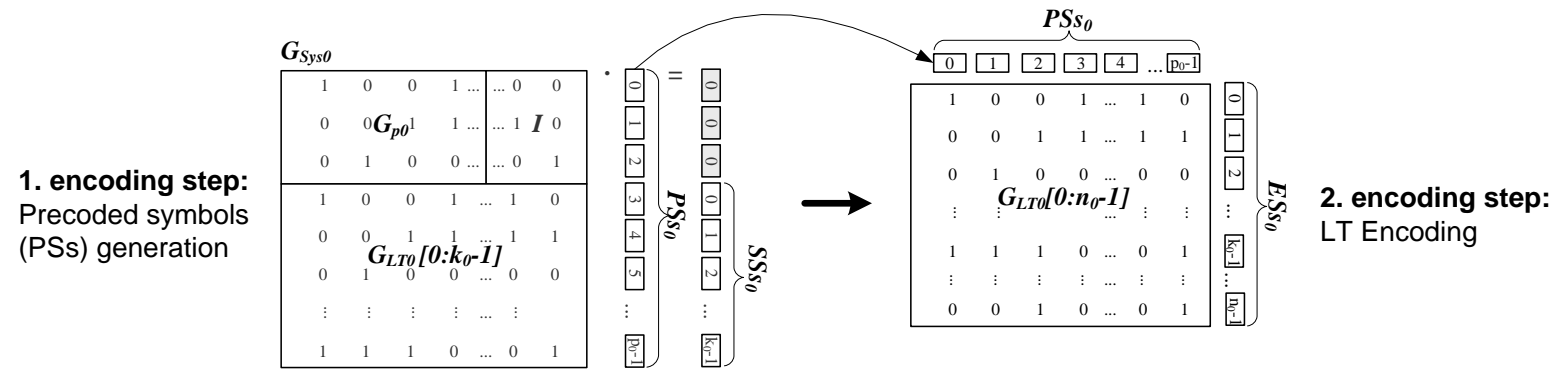

Figure 10: The Raptor encoding process for one layer $(\mathrm{L}=0)$ as specified in [10]. The first encoding step generates the precoding symbols (PSs $)_{0}$ from the source symbols $\left(\mathrm{SSs}_{0}\right)$ by use of an erasure code like LDPC. In the second step, the encoded symbols (ESs $)_{0}$ ) are generated from the PSs $\mathrm{s}_{0}$ by use of a fountain code, e.g. LT code. The code is systematic, since the first $k$ encoding symbols consist of the $k$ source symbols. 
1. encoding step:

Precoded symbols

(PSs) generation

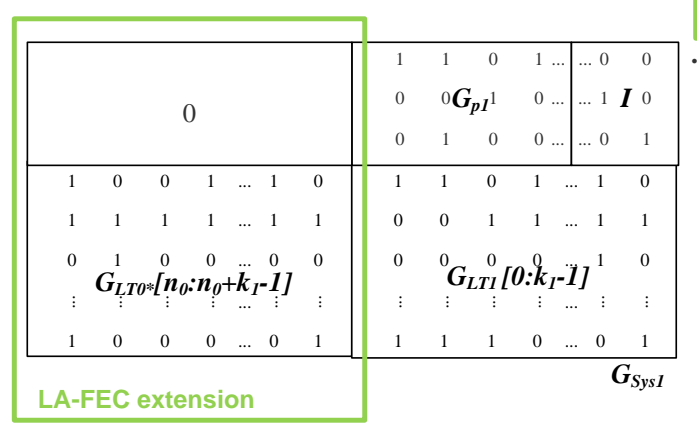

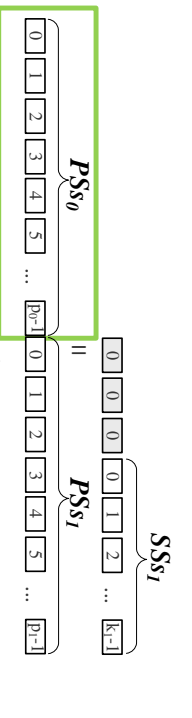

2. encoding step:

LT Encoding

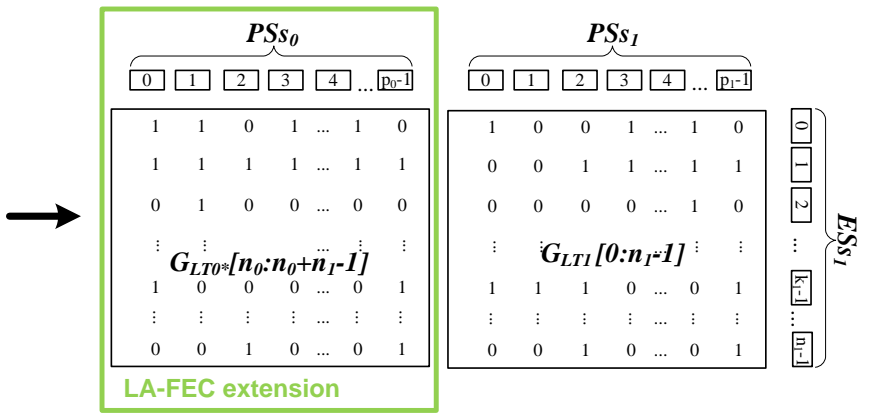

Figure 11: LA-FEC extended Raptor encoding process. The required extensions for LA-FEC are marked in green. Extending the first encoding step keeps the systematic code. The extension for the LT-Encoding connects enhancement layer to base layer. Note, the extension matrices (GLT0*) are generated by standard Raptor algorithm.

by the matrix $G_{L T O} *\left[n_{0}: n_{0}+k_{1}-1\right]$ which is the continuation of the $G_{L T O}\left[0: n_{0^{-}}-1\right]$ matrix in Figure 10. $G_{\text {Sys } 1}$ represents the first $k_{1}$ rows of the $G_{L T O} *\left[n_{0}: n_{0}+n_{1}-1\right]$ matrix in the second encoding step. Furthermore, the $P S s_{0}$ symbols from the "Layer 0" processing are included in the encoding process. These extensions assure, that the output symbols $P S s_{1}$ still lead to a systematic code after the second encoding step. The generation of the $E S s_{1}$ symbols in the second step is extended to the $P S s_{0}$ through extending the $G_{L T I}\left[0: n_{1^{-}}-1\right]$ matrix by $G_{L T O} *\left[n_{0}: n_{0}+n_{1}-1\right]$. Therefore, the $E S s_{1}$ symbols can be used in the LA-FEC together with the $E S s_{0}$ symbols for joint decoding. This is also shown in the example in Section IV. The extensions required by the LA-FEC use the algorithms for precode generation and LT Encoding as already specified in [25], leaving the specification and the defined constraints of the algorithms untouched. In case of a successful decoded "Layer 0", the introduced connections across the layers by the LA-FEC extension are not required anymore and can be removed by XORing the $P S s_{0}$ in the FEC process of "Layer 1". In such a case, "Layer 1" can be corrected following the standard Raptor coding process [26], enabling its full correction performance.

\section{Signaling and Transport of Layer-Aware FEC}

The usage of the LA-FEC in transmission systems requires specific signaling and transport techniques to support the multi-layer approach in combination with LA-FEC coding. The integration of the LA-FEC Raptor extension on link or application layer is assumed to be applied for real-time transmission over RTP [32]. For real-time applications, typically RTP is used over UDP [33] due to its connectionless and non-reliable nature it allows for minimal delay in transport. RTP provides basic features such as media synchronization, transmission order recovery, multiplexing, source identification and reception feedback information. For SVC, the RTP Payload Format for Scalable Video Coding [34] is required for media payload packetization and for MVC, the RTP Payload Format for Multiview Video Coding [35]. In particular, these payload formats for SVC and MVC define the transmission of the layered SVC and MVC data in multiple RTP sessions, which allows a transmission system using the LA-FEC coding process to simply differentiate between SVC layers and MVC views based on the transport address, such as an IP address, the UDP port or the synchronization source identifier in the RTP packet header (SSRC) [32]. Signaling of session related information is defined in the Session Description Protocol [37]. In order to signal the dependency of RTP sessions containing layers or views of the same codec, the SDP extensions in [36] are required.

For transporting the FEC coded data, the IETF created the generic FECFRAME framework defining basic means for FEC based content delivery protocols, which can be also used in RTP. This framework defines beside other features how multiple media and repair flows are treated and further provides an identification mechanism for source symbols as a part of the payload packetization information. To use this framework with the Raptor code, [38] is intended to be used.

In order to make this framework applicable to the LA-FEC the Raptor FEC scheme [39] and the Raptor RTP Payload format [40] can be used without modifications for packetizing the repair flow. The signaling for the Raptor FEC scheme is defined in [38], where the indication of depending repair flows is already defined in [41], as required for LA-FEC base layer protection and LA-FEC enhancement layer protection.

\section{Simulation Results For SVC LA-FEC AT THE APPLICATION LAYER IN DVB-H}

\section{A. Simulation Setup}

The simulations in this section are based on a mobile Broadcast scenario where two device capabilities, QVGA and VGA, are supported by a single DVB-H service using SVC (cf. Figure 12). For increasing the robustness of the whole service, the link layer FEC defined in DVB-H, MPE-FEC, and the proposed Raptor-based LA-FEC solution is evaluated on 


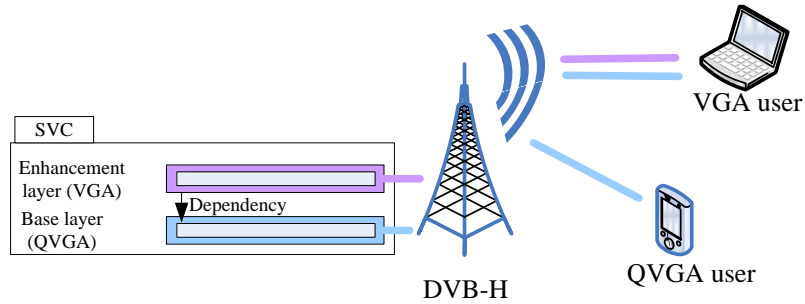

Figure 12: Support of different device capabilities (QVGA+VGA) using $\mathrm{SVC}$ in a DVB-H broadcast system.

the application layer using different code rate distributions across media coding layers.

\section{B. Channel Model}

The simulation scenario consists on a Typical Urban 6-taps (TU6) channel model with a constant Doppler (i.e., user velocity). The TU6 channel models the time variant smallscale fluctuations of the received signal due to receiver mobility (fast fading), and it was proven to be representative for DVB-H mobile reception for Doppler frequencies above $10 \mathrm{~Hz}$ (i.e., vehicular reception) [31]. We consider the DVB-H physical layer transmission mode: FFT size 8K, OFDM symbol guard interval (GI) 1/4, modulation 16-QAM and code rate $1 / 2$, which provides a channel capacity of about $10 \mathrm{Mbps}$.

\section{Media Encoding}

The video encoding was performed using the SVC reference software version JSVM 9.1. A simple rate control was employed to achieve an approximately constant service rate. The video was encoded in small chunks, where each chunk consists of a preceding IDR frame followed by three groups of picture of size 8 (GOP8), i.e. 25 frames. Each chunk was encoded multiple times with different quantization parameters (QP) values. Depending on the selected video rate, the chunk with QP value providing the target bitrate was selected and the different chunks were concatenated to one video stream. The chunk wise encoding gives a random access point (RAP) interval of 1 second at 25 frames per second (fps).

The test sequence "Soccer" ${ }^{1}$ with a duration of 10 seconds was selected for the simulations. An SVC bit stream with two scalable layers was encoded using the scalable high profile of H.264/AVC. In particular the stream contained a base layer which provides QVGA at $12.5 \mathrm{fps}$, and an enhancement layer increasing the quality to VGA at 25 fps. Freeze frame error concealment was used, where in case of frame loss, the last decoded picture is copied for output. In cases where only the enhancement layer was lost, the up-scaled picture of the QVGA layer was used for PSNR calculation. A summary of the encoding parameters can be found in Table II.

Table II: ENCODING PARAMETERS FOR SVC BITSTREAM WITH TEMPORAL AND SPATIAL SCALABILITY.

\begin{tabular}{|l|c|c|c|}
\hline & Quality & Bitrate & PSNR VGA \\
\hline H.264/AVC & QVGA & $225 \mathrm{kbps}$ & $\begin{array}{c}31.3 \mathrm{~dB} \\
\text { (upscaled) }\end{array}$ \\
Base layer & $12.5 \mathrm{fps}$ & & $35.4 \mathrm{~dB}$ \\
\hline SVC & VGA & $647 \mathrm{kbps}$ & \\
Enhancement layer & $25 \mathrm{fps}$ & & \\
\hline
\end{tabular}

\footnotetext{
${ }^{1}$ Other sequences have been tested which show a different rate-distortion performance but similar performance in the comparison of LA-FEC and STFEC.
}

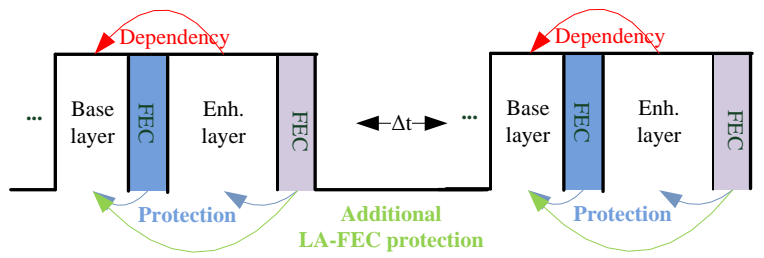

Figure 13: SVC transmission of base (QVGA@12.5fps) and enhancement layer (VGA@25fps) in different time-sliced bursts, the second immediately following the other.

\section{DVB-H Transmission Scheduling}

DVB-H applies a so called time slicing approach, where data is transmitted in bursts in order to save battery power by switching off the receiver between bursts. Therefore, the two SVC layers were transmitted in two different time-sliced bursts, the second containing the enhancement layer directly following the first containing the base layer. Figure 13 illustrates such a transmission scheduling, where the red arrows show the SVC layer coding dependencies, the black arrows the protection by ST-FEC and the green arrows the protection added by LA-FEC.

The source block size for FEC generation is aligned to the chunk size, i.e. each source block starts with an IDR RAP and incorporates all GOP8s of the chunk, equivalent to $1 \mathrm{sec}$ of media data.

\section{E. FEC Settings}

The overall service bitrate (including media data and parity bits) was fixed to $1300 \mathrm{kbps}$. The code rates for base and enhancement layer are adjusted to reach this bitrate. For example, the equal error protection (EEP) scheme allows for a code rate of 0.68 for each media layer. Furthermore, different unequal error protection (UEP) schemes were applied with either stronger protection in base layer or stronger protection in the enhancement layer. It should be noted here, that with ST-FEC, typically UEP with a stronger protection for the enhancement layer is a non reasonable setting. The selected video streams are encapsulated in RTP packets according to their specific RTP payload format [34] and the FEC symbols are encapsulated within the related RTP payload format [40] and subsequently into IP streams.

\section{F. Results}

The plots in Figure 14 show the IP packet error rate for the SVC base (left) and enhancement layer (right) and Figure 15 shows the resulting video quality in terms of average PSNR (right) and the number of freeze frames (left) over different reception conditions in terms of $\mathrm{C} / \mathrm{N}$ for the different transmission schemes. Note that there are in total 250 frames.

The IP packet error plot in Figure 14 illustrates the effect of the LA-FEC scheme compared to the ST-FEC scheme. Although the total amount of protection packets is the same for the different coding schemes, the number of lost base layer IP packets is for all code rate distributions significantly lower when applying LA-FEC. All LA-FEC schemes show a similar base layer decoding probability whereas the ST-FEC decoding probability strongly depends on the assigned code rate. As expected from the theoretical analysis in Section IV.B, the effective IP packet error rate of enhancement layer data after FEC correction is increased due to the introduced dependency 

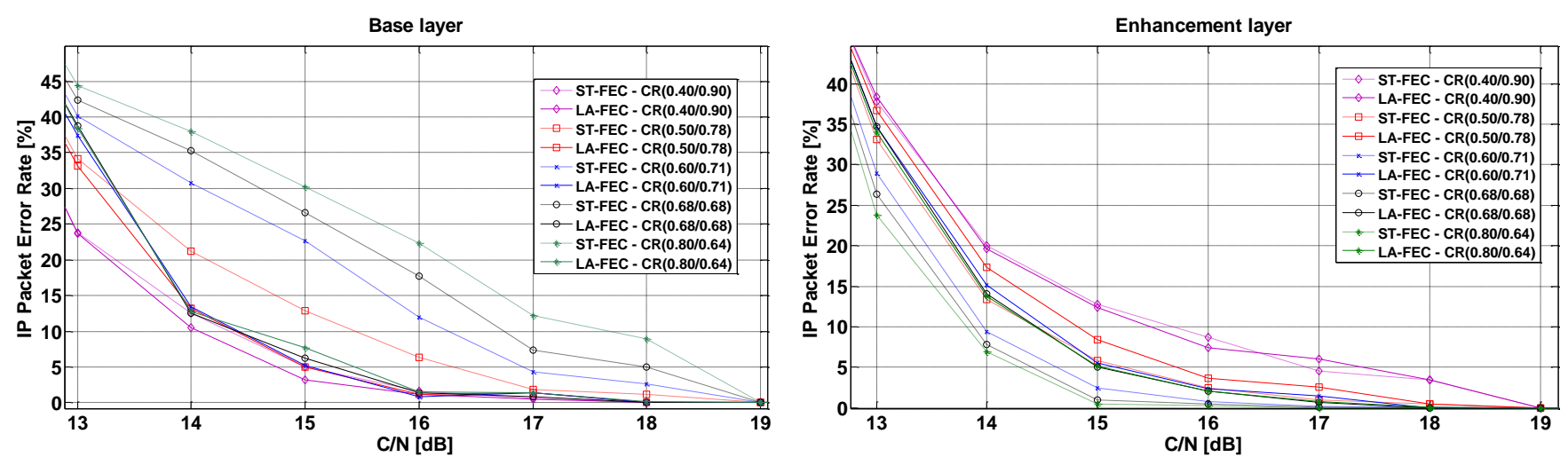

Figure 14: IP packet error rate for base (left) and enhancement layer (right) using ST-FEC and LA-FEC with different code rate distributions across SVC layers at a fixed service bitrate of $1300 \mathrm{kbps}$.

to the base layer data when applying LA-FEC. However, due to the fact that enhancement layer data is useless without the reception of the respective base layer data, the increased IP packet error rate has no negative impact on the perceived video quality.

Considering the video quality in Figure 15, all LA-FEC settings show a better performance compared to ST-FEC. The best ST-FEC scheme has a strong UEP spreading (cf. $\mathrm{CR}(0.50 / 0.78)$ in the figure). However, further increasing the base layer protection in the ST-FEC case decreases the overall performance (cf. $\mathrm{CR}(0.40 / 0.90)$ in the figure). Although there is not a significant performance difference between the tested LA-FEC settings, the best LA-FEC scheme is the one using EEP code rate setting (cf. $(\mathrm{CR}(0.68 / 0.68)$ in the figure). The LA-FEC EEP scheme outperforms the best ST-FEC scheme by approx. $2 \mathrm{~dB}$ in terms of PSNR for the $\mathrm{C} / \mathrm{N}$ value range from $14 \mathrm{~dB}$ to $16 \mathrm{~dB}$. Within this area, the video service with LA-FEC achieves a video quality in terms of PSNR over 30 $\mathrm{dB}$, which can be assumed as an acceptable quality from a users point of view. It is further interesting, that the difference in performance between the difference LA-FEC schemes is not as big as between the ST-FEC schemes. Even a stronger protection for the enhancement layer shows a relatively better performance than the ST-FEC schemes when applying LAFEC. This also allows for applying new operation points using stronger protections in the enhancement layer, which might be useful in applications such as conditional access. With LAFEC and conditional access, a service could be applied with a free base layer providing low quality and low robustness, e.g. CR 0.80 . With additionally receiving the enhancement layer of

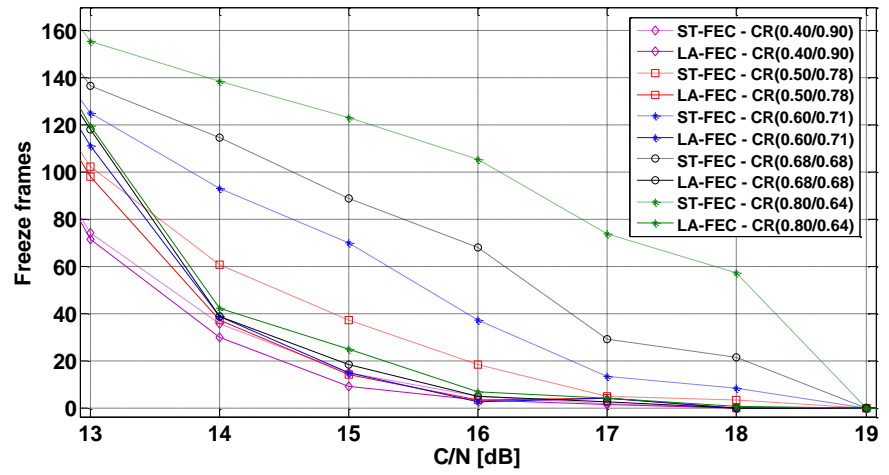

the premium service would not only increase the video quality but also the service robustness (cf. CR(0.80/0.64) in Figure 15). Taking into account that LA-FEC with EEP gives the best performance, LA-FEC eases the adjustment of the code rates for a multi layer transmission system. Considering freeze frames, LA-FEC gives a significant improvement to the number of frozen frames, which is due to the lower base layer IP packet error rate. All LA-FEC schemes show a lower number of freeze frames than the related ST-FEC scheme and all LA-FEC scheme reach at least the performance of the best ST-FEC scheme. Thereby, LA-FEC significantly increases the service reliability. This is in general interesting, since increasing the service robustness with layered-media by UEP compared to single layer typically comes at the cost of a reduced enhancement layer protection. Using LA-FEC allows to achieve a similar effect without reducing the enhancement layer robustness (cf. CR(0.68/0.68) in Figure 15).

\section{CONCLUSION}

In this work, we present the layer-aware FEC (LA-FEC) concept for improving performance in broadcast of multilayered media. The LA-FEC concept can be implemented either on the physical or any upper communication system layer. We present a theoretical model, which shows the gain introduced by LA-FEC compared to standard FEC schemes. We described the application of the LA-FEC concept to the Raptor code at the link or application layer. We further describe the application means for transport and signaling of LA-FEC. Simulations results for application layer LA-FEC in

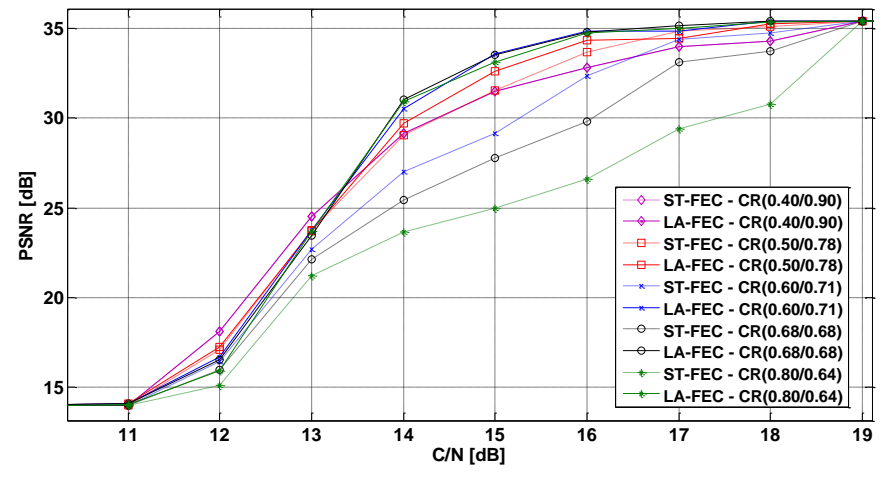

Figure 15: Average number of freeze frames of 250 frames (left) and the average PSNR value (right) for a VGA receiver using ST-FEC and LA-FEC with different code rate distributions across SVC layers at a fixed service bitrate of $1300 \mathrm{kbps}$. 
a DVB-H scenario showed that the theoretical gain can also be translated to a real channel. Future work will be the investigation of the performance of LA-FEC on physical layer. We further plan to analyze the performance of combinations of LA-FEC with related schemes.

\section{REFERENCES}

[1] H. Schwarz, D. Marpe, and T. Wiegand, "Overview of the scalable video coding extension of the H.264/AVC standard," IEEE Trans. Circuits and Systemd for Video Technology, Vol. 17, Issue 9, pp. 11031120, September 2007.

[2] P. Merkle, Y. Morvan, A. Smolic, D. Farin, K. Müller, P.H.N. de With, and T. Wiegand, "The effects of multiview depth video compression on multiview rendering," Signal Processing: Image Communication special issue on advances in three-dimensional television and video, Vol. 24, Issues 1-2, pp. 73-88, January 2009.

[3] DVB TM-H NGH, "Call for Technologies (CfT) v 1.0," DVB TM 4270r2, November 2009.

[4] ATSC, "ATSC-Mobile DTV Standard, Part 7 - AVC and SVC Video System Characteristics," Document A/153 Part 7:2009, October 2009.

[5] ETSI TR 102993 V1.1.1, "Digital Video Broadcasting (DVB); Upper Layer FEC for DVB Systems," February 2011.

[6] D. J. C. MacKay and R. M. Neal, "Near Shannon limit performance of low density parity check codes", Electron Letters, Vol. 33, Issue 6, pp. 457-458, March 1997.

[7] C. Berrou, "The ten-year-old turbo codes are entering into service," IEEE Communications Magazine, Vol. 41, Issue 8, pp. 110-116, August 2003.

[8] A. Shokrollahi, "Raptor Codes," IEEE Transactions on Information Theory, Vol. 52, Issue 6, pp. 2551-2567, 2006.

[9] M. Luby, A. Shokrollahi, M. Watson, T. Stockhammer, L. Minder, "RaptorQ Forward Error Correction Scheme for Object Delivery," IETF RMT, draft-ietf-rmt-bb-fec-raptorq-04, August 2010, http://tools.ietf.org/html/draft-ietf-rmt-bb-fec-raptorq-04.

[10] ETSI TS 102472 v1.3.1, "Digital Video Broadcast (DVB); IP Datacast over DVB-H: Content Delivery Protocols", June 2009.

[11] ETSI TS 102584 v1.2.1, "Digital Video Broadcasting (DVB); DVB-SH Implementation Guidelines", January 2011.

[12] 3GPP TS 26.346, "Technical specification group services and system aspects; multimedia broadcast/multicast service (MBMS); Protocols and codecs".

[13] C. Hellge, S. Mirta, T. Schierl, and T. Wiegand, "Mobile TV with SVC and Hierarchical Modulation for DVB-H Broadcast Services," IEEE International Symposium on Broadband Multimedia Systems and Broadcasting (BMSB), Bilbao, Spain, May 2009.

[14] S. Mirta, et al., "HD Video Broadcasting using Scalable Video Coding combined with DVB-S2 Variable Coding and Modulation," ASMA and SPSC, September 2010.

[15] B. Masnick and J. Wolf, "On linear unequal error protection codes," IEEE Transactions on Information Theory, Vol. 13, Issue 4, pp. 600607, 1967.

[16] C. C. Kilgus and W. C. Gore, "A class of cyclic unequal-error-protection codes," IEEE Transaction on Information Theory, Vol. 18, Issue 5, pp. 687-690, September 1972.

[17] I. Boyarinov and G. Katsman, "Linear unequal error protection codes," Information Theory, IEEE Transactions on, Vol. 27, Issue 2, pp. 168$175,1981$.

[18] N. Rahnavard and F. Fekri, "New results on unequal error protection using LDPC codes," IEEE Communications Letters, Vol. 10, Issue 1, pp. 43-45, 2006.

[19] N. Rahnavard, B. N. Vellambi, and F. Fekri, "Rateless Codes With Unequal Error Protection Property," IEEE Transactions on Information Theory, Vol. 53, Issue 4, pp. 1521-1532, 2007.

[20] A. Bouabdallah and J. Lacan, "Dependency-aware unequal erasure protection codes," Journal of Zhejiang University SCIENCE A 2006 7(Suppl. I):27-33, 2006.

[21] M. Bogino, P. Cataldi, M. Grangetto, E. Magli and G. Olmo, "SlidingWindow Digital Fountain Codes for Streaming of Multimedia Contents," ISCAS 2007, USA, May 2007.

[22] D. Sejdinovic, D. Vukobratovic, A. Doufexi, V. Senk, und R. Piechocki, "Expanding window fountain codes for unequal error protection," IEEE
Transactions on Communications, Vol. 57, Issue 9, pp. 2510-2516, 2009.

[23] D. Vukobratovic, V. Stankovic, D. Sejdinovic, L. Stankovic, Z. Xiong, "Scalable video multicast using expanding window fountain codes," IEEE transaction on Multimedia, Vol. 11, Issue 6, pp. 1094-1104, 2009.

[24] OSI Reference Model, Hubert Zimmermann, IEEE Transactions on Communications, Vol. COMM-28(4), p425, April 1980.

[25] M. Luby, "LT-Codes," in Proceedings of the ACM Symposium on Foundation of Computer Science (FOCS), 2002.

[26] M. Luby, A. Shokrollahi, M. Watson, T. Stockhammer, "Raptor Forward Error Correction Scheme for Object Delivery," IETF RFC 5053, Internet Engineering Task Force (IETF), Network Working Group, September 2007, http://tools.ietf.org/html/rfc5053.

[27] C. Hellge, T. Schierl, and T. Wiegand, "Mobile TV using scalable video voding and layer-aware forward error correction," IEEE International Conference on Multimedia and Expo (ICME'08), Hanover, Germany, June 2008.

[28] C. Hellge, T. Schierl, and T. Wiegand, "Multidimensional Layered Forward Error Correction Using Rateless Codes," IEEE International conference on communication (ICC), Bejing, China, 2008.

[29] C. Hellge, T. Schierl, and T. Wiegand, "Receiver driven layered multicast with layer-aware forward error correction," Proceedings of ICIP 2008, pp. 2304-2307, 2008

[30] C. Hellge, D. Gomez-Barquero, T. Schierl, and T. Wiegand, "Intra-burst Layer Aware FEC for Scalable Video Coding Delivery in DVB-H," Proceedings of ICME 2010, Singapur, Singapur, July 2010.

[31] G. Faria, J. A. Henriksson, E. Stare, and P. Talmola, "DVB-H: Digital Broadcast Services to Handheld Devices," Proc of the IEEE, Vol. 94, Issue 1, pp. 194-209, January 2006.

[32] H. Schulzrinne, S. Casner, R. Frederick, and V. Jacobson, "RTP: A Transport Protocol for Real-time Applications", IETF STD 0064, RFC 3550, July 2003, http://tools.ietf.org/html/rfc3550.

[33] J. Postel, "User Datagram Protocol", IETF STD 6, RFC 768, September 1981, http://tools.ietf.org/html/rfc768.

[34] S. Wenger, Y.-K. Wang, T. Schierl, and A. Eleftheriadis, "RTP payload format for SVC video", work in progress, Internet Engineering Task Force (IETF), Audio Video Transport Group (avt), draft-ietf-avt-rtp-svc21, April 2010, http://tools.ietf.org/html/draft-ietf-avt-rtp-svc.

[35] Y.-K. Wang, T. Schierl, "RTP Payload Format for MVC Video," work in progress, IETF AVT, draft-ietf-avt-rtp-mvc-01.txt, October 2010, http://tools.ietf.org/html/draft-ietf-avt-rtp-mvc-01.

[36] T. Schierl and S. Wenger, "Signaling media decoding dependency in Session Description Protocol (SDP)", IETF MMUSIC, April 2009, http://tools.ietf.org/html//rfc5583.

[37] M. Handly, V. Jacobson, and C. Perkins, "SDP: Session Description Protocol", IETF RFC 4566, July 2006, http://tools.ietf.org/html/rfc4566.

[38] M. Watson, "Forward Error Correction (FEC) Framework", IETF FECFRAME draft-ietf-fecframe-framework-09, July 2010, http://tools.ietf.org/html/draft-ietf-fecframe-framework.

[39] M. Watson, "Raptor FEC Schemes for FECFRAME", IETF FECFRAME, draft-ietf-fecframe-raptor-02, March 2010, http://tools.ietf.org/html/ draft-ietf-fecframe-raptor.

[40] M. Watson, "RTP Payload Format for Raptor FEC", IETF FECFRAME, draft-ietf-fecframe-rtp-raptor-03, March 2010, http://tools.ietf.org/html/ draft-ietf-fecframe-rtp-raptor.

[41] A. Begen, "SDP Elements for FEC Framework", IETF FECFRAME, draft-ietf-fecframe-sdp-elements-07, July 2010, http://tools.ietf.org/html/draft-ietf-fecframe-sdp-elements-04.

[42] DVB TM-H, "Response to DVB-NGH Call for Technologies, LayerAware FEC for Layered Media Transmission," TM-NGH092, February 2010.

[43] T. Wiegand, G.J. Sullivan, G. Bjontegaard, and A. Luthra, "Overview of the H.264/AVC Video Coding Standard," IEEE Transactions on Circuits and Systems for Video Technology, Vol. 13, Issue 7, pp. 560-576, July 2003.

[44] ETSI EN 302583 V1.1.2, "Digital Video Broadcasting (DVB); Framing Structure, channel coding and modulation for Satellite Services to Handheld devices (SH) below $3 \mathrm{GHz}, "$ February 2010.

[45] ETSI EN 302755 V1.1.1, "Digital Video Broadcasting (DVB); Frame structure channel coding and modulation for a second generation digital terrestrial television broadcasting system (DVB-T2)," September 2009.

[46] C. Berrou, "The ten-year-old turbo codes are entering into service," IEEE Communications Magazine, Vol. 41, Issue 8, pp. 110-116, August 2003. 


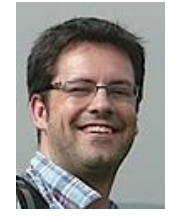

Cornelius Hellge received the Dipl.-Ing. degree (M.Sc.) in Media Technology from the Ilmenau University of Technology, Germany in July 2006. He has been with Fraunhofer HHI since 2005. Since 2009, he works in parallel as a researcher in the Image Communication Laboratory at the Berlin Institute of Technology. He contributed to different standardization bodies (e.g., DVB, 3GPP) and has done different research works on reliable real-time transmission of H.264/AVC and Scalable Video Coding (SVC) in mobile point-to-point, multicast, broadcast, IPTV, and P2P environments. His main research interests are in the area of reliable video transmission and signaling, in particular in forward error correction and in the joint use of channel and source coding. Currently, he is working on media delivery in CDNs in the context of the European research project of the FP7 program OCEAN. He was a Co-editor of the DVB bluebook on upper layer forward error correction. Currently, he is actively participating in the standardization process of the next generation handheld standard DVB-NGH and the 3GPP SA4 group.

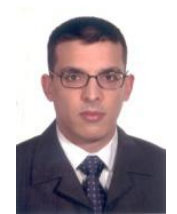

David Gómez-Barquero is a post-doc guest researcher at the Fraunhofer Heinrich Herzt Institute (HHI) in Berlin, Germany. He received a double M.Sc. degree in Telecommunications engineering from the Universidad Politécnica de Valencia (UPV), Spain, and the University of Gavle, Sweden, in 2004; and a Ph.D. in Telecommunications from UPV in 2009. During his doctoral studies he was a guest researcher at the Royal Institute of Technology, Sweden, the University of Turku, Finland, and the Technical University of Braunschweig, Germany. $\mathrm{He}$ also did an internship at Ericsson Eurolab, Aachen, Germany. His main research interests are in the area of mobile multimedia broadcasting, in particular radio resource management, forward error correction, and network planning issues in both DVB and 3GPP MBMS systems. Dr. Gómez-Barquero chaired the special interest group on hybrid cellular and broadcasting networks in the European COST2100 cooperation action, and co-edited the DVB bluebook on upper layer forward error correction as an invited expert. Currently, he is actively participating in the standardization process of the next generation handheld mobile broadcasting standard DVB-NGH.

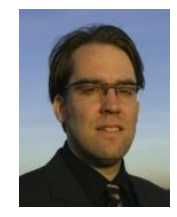

Thomas Schierl (M'07) received the Dipl.-Ing. (M.Sc.) degree in Computer Engineering from the Berlin Institute of Technology, Germany in December 2003 and Dr.-Ing. $(\mathrm{Ph} . \mathrm{D})$ degree in Electrical Engineering and Computer Science from Berlin Institute of Technology in October 2010. Since 2010, Thomas is head of the Multimedia Communications Group in the Image Processing Department of Fraunhofer HHI, Berlin. Since September 2004, Thomas is a Senior Researcher in the Image processing department, where Thomas is responsible as a project manager for various scientific as well as Industry-funded research projects. Thomas is the co-author of various IETF RFCs, beside others he is author of the IETF RTP Payload Format for H.264 SVC (Scalable Video Coding), the IETF RTP Payload for Multi View Coding (MVC) as well as of the IETF RFC on Rapid Synchronization of RTP flows (RFC 6051). In the ISO/IEC MPEG group, Thomas is co-editor of the MPEG Standard on Transport of H.264 SVC as well as H.264 MVC over MPEG-2 Transport Stream. Thomas is also a co-editor of the AVC File Format. Typically, he is participating MPEG, IETF, 3GPP and DVB meetings.

In 2007, he visited the Image, Video, and Multimedia Systems group of Prof. Bernd Girod at Stanford University, CA, USA for different research activities. Thomas' research interests include real-time media delivery over IP networks, which may be mobile or wired, with or without infrastructure (e.g., Mobile Ad Hoc Networks (MANETs)), or based on traditional as well as on overlay/Peer-to-Peer (P2P) mobile IPTV approaches. Currently, Thomas is working on mobile media content delivery over HTTP.

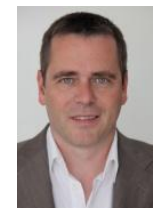

Thomas Wiegand (M'05, SM'08, F'11) is a professor at the department of Electrical Engineering and Computer Science at the Berlin Institute of Technology, chairing the Image Communication Laboratory, and is jointly heading the Image Processing department of the Fraunhofer Institute for Telecommunications - Heinrich Hertz Institute, Berlin, Germany. He received the Dipl.-Ing. degree in Electrical Engineering from the Technical University of Hamburg-Harburg, Germany, in 1995 and the Dr.-Ing. degree from the University of Erlangen-Nuremberg, Germany, in 2000. He joined the Heinrich Hertz Institute in 2000 as the head of the Image Communication group in the Image Processing department. His research interests include video processing and coding, multimedia transmission, as well as computer vision and graphics.

From 1993 to 1994, he was a Visiting Researcher at Kobe University, Japan. In 1995, he was a Visiting Scholar at the University of California at Santa Barbara, USA. From 1997 to 1998, he was a Visiting Researcher at Stanford University, USA and served as a consultant to 8x8, Inc., Santa Clara, CA, USA. From 2006-2008, he was a consultant to Stream Processors, Inc., Sunnyvale, CA, USA. From 2007-2009, he was a consultant to Skyfire, Inc., Mountain View, CA, USA. Since 2006, he has been a member of the technical advisory board of Vidyo, Inc., Hackensack, NJ, USA.

Since 1995, he has been an active participant in standardization for multimedia with successful submissions to ITU-T VCEG, ISO/IEC MPEG, 3GPP, DVB, and IETF. In October 2000, he was appointed as the Associated Rapporteur of ITU-T VCEG. In December 2001, he was appointed as the Associated Rapporteur / Co-Chair of the JVT. In February 2002, he was appointed as the Editor of the H.264/MPEG-4 AVC video coding standard and its extensions (FRExt and SVC). From 2005-2009, he was Co-Chair of MPEG Video.

In 1998, he received the SPIE VCIP Best Student Paper Award. In 2004, he received the Fraunhofer Award and the ITG Award of the German Society for Information Technology. The projects that he co-chaired for development of the H.264/MPEG-4 AVC standard have been recognized by the 2008 ATAS Primetime Emmy Engineering Award and a pair of NATAS Technology \& Engineering Emmy Awards. In 2009, he received the Innovations Award of the Vodafone Foundation, the EURASIP Group Technical Achievement Award and the Best Paper Award of IEEE Transactions on Circuits and Systems for Video Technology. In 2010, he received the Eduard Rhein Technology Award. Professor Wiegand was elected Fellow of the IEEE in 2011.

He was a Guest Editor for the IEEE Transactions on Circuits and Systems for Video Technology for its Special Issue on the H.264/AVC Video Coding Standard in July 2003, its Special Issue on Scalable Video CodingStandardization and Beyond in September 2007, and its Special Section on the Joint Call for Proposals on High Efficiency Video Coding (HEVC) Standardization. Since January 2006, he has been an Associate Editor of IEEE Transactions on Circuits and Systems for Video Technology. 